\title{
CONCEPÇÃO DE UM MODELO DE INDICADORES DE GESTÃO DE PESSOAS: O CASO DOS INSTITUTOS FEDERAIS DE EDUCAÇÃO, CIÊNCIA E TECNOLOGIA
}

THE MODEL DEVELOPMENT OF HUMAN RESOURCE MANAGEMENT INDICATORS: THE CASE OF THE FEDERAL INSTITUTES OF EDUCATION, SCIENCE AND TECHNOLOGY

Eduardo Rocha Bassi

Instituto Federal do Rio Grande do Sul, Santa Maria, RS, Brasil. E-mail: eduardo.rbassi@hotmail.com

Eugênio de Oliveira Simonetto Universidade Federal de Santa Maria, Santa Maria, RS, Brasil. E-mail: eosimonetto@ufsm.br

Recebido em: 02.06.2017- Aceito em: 08.08.2017

\section{RESUMO}

O presente estudo teve como objetivo geral desenvolver um modelo de Indicadores de Gestão de Pessoas para Institutos Federais. Inicialmente, foram coletados indicadores em 228 relatórios de gestão, extraídos do banco de dados do TCU e tratados por meio de análise de conteúdo. Em seguida, formatou-se um modelo composto por nove Indicadores de Gestão de Pessoas, coletados e ponderados por ordem de preferência (aplicação prática) e respaldo teórico (análise bibliográfica). O referido modelo foi avaliado por meio de entrevistas com gestores de pessoal de três Institutos Federais. Os entrevistados consideram que quase todos os indicadores apresentados possuem aplicação prática e que o modelo pode contribuir com a tomada de decisões gerenciais e facilitar a transparência de informações à sociedade e aos órgãos controladores.

PALAVRAS-CHAVE: Indicadores; Gestão de Pessoas; Institutos Federais de Educação, Ciência e Tecnologia.

\section{ABSTRACT}

This study aimed to develop a model of Human Resource Management Indicators for the Federal Institutes. Initially, indicators were collected from 228 management reports, obtained from the TCU database, and this data was then processed through a content analysis. After this, it was established a model consisting of nine indicators of Human Resources, which were collected and considered in order of preference (practical application) and theoretical support (literature review). The proposed model was evaluated through interviews with human resources mana- 
gers of three Federal Institutes. The respondents believe that almost all indicators presented have practical application, and that the model can contribute to the managerial decision-making and facilitate the transparency of information to society and to the controlling bodies.

KEYWORDS: Indicators; Human resource management; Federal Institutes of Education, Science and Technology.

\section{INTRODUÇÃO}

As organizações, em sua maioria, utilizam os mais diversos e variados tipos de métodos de medição para aferir o seu desempenho ou avaliar o seu desenvolvimento organizacional, incluindo, principalmente, os indicadores de gestão. O principal objetivo da utilização de indicadores consiste em fornecer parâmetros que deem maior sustentação à tomada de decisões dos gestores de uma organização (Amato Neto, 2009; Takashina e Flores, 1996). Dessa forma, pode-se considerar que a principal função dos indicadores de gestão, seja através da mediação de desempenho, seja através de resultados, é a de auxiliar os gestores em processos decisórios.

Com relação aos órgãos e instituições públicas brasileiras, além da necessidade da aplicação de indicadores de gestão para o apoio à tomada de decisão, tais organizações também devem utilizá-los para prestar contas à sociedade e aos órgãos de controle. Isso porque, conforme as últimas normativas publicadas para prestação de contas anual, o Tribunal de Contas da União (TCU), órgão controlador que julga as contas de administradores públicos, vem questionando essas organizações sobre o uso de métodos de medição e aferição de resultados.

A importância da utilização desses métodos de mensuração ampliou-se ainda mais nos últimos anos, pois um dos principais investimentos realizados pelo governo federal brasileiro foi a expansão da Rede Federal de Educação Profissional, Científica e Tecnológica, que possibilitou o ingresso de milhares de novos servidores públicos vinculados ao Ministério da Educação (MEC). Todo esse investimento repercutiu diretamente em maiores gastos e investimentos com pessoal, envolvendo processos de seleção, ambientação, remuneração, capacitação, qualificação, entre outros.

Em consequência, com o intuito de monitorar processos envolvendo o capital humano, as instituições envolvidas necessitam adotar sistemas para medição e comparação de informações acerca das ações da gestão de pessoas, isto é, demandam a utilização de Indicadores de Gestão de Pessoas. Segundo Pomi (2002), a mensuração de informações e resultados, no campo dos Recursos Hu- 
manos $(\mathrm{RH})$, permite aos gestores um maior conhecimento do desempenho da gestão de pessoas na organização, bem como possibilita comparar resultados, identificar pontos de melhoria e subsidiar ações corretivas.

Os Institutos Federais de Educação, Ciência e Tecnologia (IFETs) estabelecidos pela Lei $n^{\circ}$ 11.892, de 29 de dezembro de 2008, que instituiu a Rede Federal de Educação Profissional, Científica e Tecnológica (EPCT), do mesmo modo que todos os demais órgãos e instituições públicas federais, emitem, anualmente, um Relatório de Gestão, apresentando ao Tribunal de Contas da União documentos, informações e demonstrativos de natureza contábil, financeira, orçamentária, operacional e patrimonial, incluindo, também, um subitem específico acerca de "Indicadores Gerenciais sobre Recursos Humanos", previsto nas normativas do TCU, as quais dispõem sobre orientações quanto à elaboração de conteúdos dos relatórios de gestão.

Devido à recente fase de crescimento e expansão da rede, os IFETs vêm apresentando uma preocupação cada vez maior sobre o controle dos recursos físicos, humanos e financeiros, para que a ampliação da oferta educacional ocorra sem prejuízo da qualidade. Dessa maneira, uma das preocupações latentes, e de grande impacto operacional e orçamentário para os institutos, diz respeito ao sistema de medição e controle de informações da gestão de pessoas, que está relacionado diretamente com as atuais demandas do TCU.

Entretanto, no que diz respeito a esses indicadores da área de gestão de pessoas, cobrados anualmente pelos órgãos controladores, ainda não existem indicadores pré-estabelecidos para aplicação. Atualmente, os IFETs buscam, por conta própria, desenvolver e utilizar tais indicadores, pois não contam com um modelo de Indicadores de Gestão de Pessoas proposto pelo TCU ou pela Secretaria de Educação Profissional e Tecnológica/MEC.

Além disso, Bergue (2014) argumenta que os indicadores de gestão ainda não são empregados com a merecida intensidade na administração pública, visto que, atualmente, a utilização desses indicadores limita-se a aspectos orçamentários e financeiros apenas e, ainda assim, por imposição legal.Desse modo, o presente estudo foi orientado pelo seguinte problema de pesquisa: quais são os Indicadores de Gestão de Pessoas que poderiam ser aplicados pelos IFETs?

O objetivo geral da pesquisa consiste em desenvolver um modelo de Indicadores de Gestão de Pessoas para IFETs, de forma a contribuir com a tomada de decisões gerenciais e facilitar a transparência de informações à sociedade e aos órgãos controladores. O trabalho está organizado da seguinte forma: na seção 2 , são apresentados os indicadores aplicados à gestão de pessoas; na seção 3, é apresentado 
o método utilizado no desenvolvimento da pesquisa; na seção 4, apresentam-se os resultados da pesquisa e, por fim, na seção 5,as considerações finais do artigo.

\section{INDICADORES DE GESTÃO DE PESSOAS}

De acordo com Le Gall (2008), a área de gestão de pessoas é "[...] a que mais evoluiu desde os anos 1960 e ainda mais rápido desde a década de 1980, com a ênfase nos recursos humanos". A Gestão de Pessoas - ou área de Recursos Humanos - caracteriza-se como um conjunto de políticas e práticas que permitem a conciliação de expectativas entre a organização e os seus colaboradores.

No setor público, as ações da área de gestão de pessoas podem ser entendidas como esforços orientados para a provisão, manutenção e desenvolvimento de pessoas nas organizações públicas Bergue (2010). Essas ações são representadas por um conjunto de atividades e devem ser monitoradas e controladas por meio da medição de indicadores Assis (2012).

Assis (2012) entende que as medições da área de gestão de pessoal são fundamentais para se determinar um ponto de chegada, estabelecer parâmetros, entender e compartilhar resultados, determinar e mobilizar recursos, corrigir rumos, reforçar ações e recompensar resultados.

Embora a importância do uso dos Indicadores de Gestão de Pessoas não seja uma novidade para boa parte das organizações, alguns fatores atuais, como a necessidade de otimização dos processos para a redução de custos, têm impulsionado as organizações e seus gestores para uma maior utilização de indicadores envolvendo a área de recursos humanos (Carlson e Kavanagh, 2012).

Com base nos indicadores estudados durante a pesquisa bibliográfica deste estudo, formatou-se um modelo teórico inicial de referência, o qual é apresentado no quadro 1.

\begin{tabular}{|c|c|}
\hline Modelo teórico inicial & $\begin{array}{c}\text { Descrição dos tipos de indicadores } \\
\text { Absenteísmo }\end{array}$ \\
\hline $\begin{array}{c}\text { Compreende índices que visam demonstrar o nível de } \\
\text { abstenção do colaborador ao trabalho, podendo-se considerar } \\
\text { as faltas não justificadas, número de dias não trabalhados, } \\
\text { número de horas não trabalhadas, etc. }\end{array}$ \\
\hline $\begin{array}{c}\text { Acidentes e doenças } \\
\text { ocupacionais }\end{array}$ & $\begin{array}{c}\text { Contempla indicadores que monitoram a frequência de } \\
\text { acidentes no local de trabalho, o número de ocorrências } \\
\text { de licenças por conta de doenças ocupacionais, a taxa de } \\
\text { gravidade de acidentes, etc. }\end{array}$ \\
\hline
\end{tabular}




\begin{tabular}{|c|c|}
\hline Afastamento & $\begin{array}{c}\text { Considera os quantitativos relacionados aos afastamentos } \\
\text { legais do trabalho, incluindo licenças concedidas e demais } \\
\text { concessões de afastamentos. }\end{array}$ \\
\hline Aposentadoria & $\begin{array}{c}\text { De maneira geral, compreende índices ligados à previsão } \\
\text { de aposentadorias e à reposição de vagas oriundas de } \\
\text { aposentadorias. }\end{array}$ \\
\hline Idade e antiguidade & $\begin{array}{c}\text { Calculam o perfil etário médio dos colaboradores e a } \\
\text { antiguidade média do capital humano da organização. }\end{array}$ \\
\hline Força de trabalho & $\begin{array}{c}\text { Contempla indicadores relacionados com os quantitativos } \\
\text { de colaboradores, aumento ou redução da força de trabalho, } \\
\text { proporção de colaboradores por carreira/cargo/função, etc. }\end{array}$ \\
\hline Qualificação & $\begin{array}{c}\text { Envolve métricas que visam medir os níveis de qualificação ou } \\
\text { instrução dos colaboradores, como, por exemplo, o nível de } \\
\text { titulação acadêmica. }\end{array}$ \\
\hline Remuneração & $\begin{array}{c}\text { Diz respeito aos indicadores de gastos com pessoal, incluindo } \\
\text { remuneração média percebida, composição da folha de } \\
\text { pagamento, etc. }\end{array}$ \\
\hline Treinamento (ou \\
Capacitação) & $\begin{array}{c}\text { Compreende índices relacionados com o "giro" de } \\
\text { colaboradores (entradas e saídas), processos de admissões/ } \\
\text { contratações, demissões/desligamentos, transferências e } \\
\text { demais movimentações no quadro de colaboradores. }\end{array}$ \\
\hline $\begin{array}{c}\text { Abrange indicadores que monitoram os índices de cursos/ } \\
\text { treinamentos/capacitações realizados pelos colaboradores, os } \\
\text { valores gastos/custos, o número de horas cursadas/ofertadas, } \\
\text { o número de colaboradores capacitados, etc. }\end{array}$ \\
\hline
\end{tabular}

Quadro 1 - Modelo teórico inicial de Indicadores de Gestão de Pessoas

Fonte: Assis (2012); Bergue (2010), Caldeira (2012); Costa (2010); Fitz-enz (1984);

Jaramillo (2003); Le Gall (2008); Milioni (2005); Pomi (2002); Tachizawa et al. (2006).

O "Modelo teórico inicial de referência", apresentado no quadro 1, foi elaborado de acordo com os dez principais tipos de Indicadores de Gestão de Pessoas estudados, segundo os autores citados. Ressalta-se que se consideraram apenas os indicadores que sugerem uma base de cálculo composta exclusivamente por dados pertinentes à área de recursos humanos.

\subsection{Indicadores de gestão demandados pelo TCU}

Conforme informações consultadas no portal eletrônico do TCU, este é um tribunal administrativo que julga as contas de administradores públicos e demais responsáveis por dinheiro, bens e valores públicos federais. Anualmente, o tribunal publica algumas normativas acerca da construção dos relatórios de gestão, com o intuito de padronizar e subsidiar a elaboração desse documento que compõe o processo de contas. De acordo com Chaves (2011), o Relatório de Gestão consiste no conjunto de documentos, informações e demonstrativos de natureza 
contábil, financeira, orçamentária, operacional ou patrimonial, relativos à gestão organizacional, constituído de forma a possibilitar a visão sistêmica da conformidade e do desempenho da gestão.

A Decisão Normativa do TCU n 134/2013 (TCU, 2014), por exemplo, bem como algumas das demais normativas anteriores, determina que alguns indicadores de gestão sejam apresentados no relatório. No que se refere aos Indicadores de Gestão de Pessoas, estes são solicitados de maneira bastante flexível, isto é, o tribunal não define quais tipos de indicadores devem ser aplicados pelas instituições públicas, deixando, nesse sentido, uma lacuna a ser preenchida.

\section{MÉTODO DE TRABALHO}

O presente estudo caracteriza-se como descritivo, prescritivo e de natureza qualitativa, e a estratégia de pesquisa utilizada foi o método de estudo de caso Yin (2015), com "múltiplas unidades para análise" Gil (2010). Com o intuito de solucionar a problemática de pesquisa e atingir o objetivo proposto, este estudo foi realizado por meio de cinco etapas de trabalho, as quais podem ser visualizadas no quadro 2.

\begin{tabular}{|c|c|}
\hline Etapas de trabalho & Detalhamento \\
\hline $\begin{array}{l}\text { i. Coleta e análise dos } \\
\text { dados bibliográficos }\end{array}$ & $\begin{array}{l}\text { Coleta de dados e informações referentes à definição, caracteri- } \\
\text { zação e utilização de indicadores de gestão e também quanto aos } \\
\text { principais indicadores específicos da área de Gestão de Pessoas. }\end{array}$ \\
\hline $\begin{array}{l}\text { ii. Coleta e } \\
\text { análise dos dados } \\
\text { documentais }\end{array}$ & $\begin{array}{l}\text { Levantamento de dados sobre os Indicadores de Gestão de Pes- } \\
\text { soas aplicados e apresentados pelos Institutos Federais no perí- } \\
\text { odo de } 2009 \text { a 2014; verificação da atual situação dos IFETs no } \\
\text { processo de aplicação desses indicadores; e análise das princi- } \\
\text { pais dificuldades encontradas para a utilização de indicadores da } \\
\text { área de Gestão de Pessoas. }\end{array}$ \\
\hline $\begin{array}{l}\text { iii. Formatação } \\
\text { do modelo de } \\
\text { Indicadores de } \\
\text { Gestão de Pessoas }\end{array}$ & $\begin{array}{l}\text { Desenvolvimento de um modelo de indicadores, baseado no } \\
\text { quadro teórico inicial de referência, a partir da atual aplicação } \\
\text { prática dos Indicadores de Gestão de Pessoas de maior prefe- } \\
\text { rência para os Institutos Federais. }\end{array}$ \\
\hline $\begin{array}{l}\text { iv. Coleta e análise } \\
\text { dos dados de campo }\end{array}$ & $\begin{array}{l}\text { Realização de entrevistas com gestores de pessoal de três IFETs } \\
\text { para o levantamento e análise dos dados de campo, complemen- } \\
\text { tando as análises dos dados documentais e avaliando o modelo de } \\
\text { Indicadores de Gestão de Pessoas desenvolvido na etapa anterior. }\end{array}$ \\
\hline $\begin{array}{l}\text { v. Avaliação } \\
\text { do modelo de } \\
\text { Indicadores de } \\
\text { Gestão de Pessoas }\end{array}$ & $\begin{array}{l}\text { Análise e interpretação dos resultados da pesquisa de campo, } \\
\text { verificando se os indicadores apresentados atendem aos requi- } \\
\text { sitos essenciais recomendados e se a aplicação do modelo de- } \\
\text { senvolvido pode contribuir com a tomada de decisões gerenciais } \\
\text { e facilitar a transparência de informações à sociedade e aos ór- } \\
\text { gãos controladores. }\end{array}$ \\
\hline
\end{tabular}

Fonte: elaborado pelos autores (2016). 
Portanto, os dados secundários foram coletados mediante pesquisa bibliográfica e documental, e os dados primários coletados por meio de pesquisa de campo.

\subsection{Pesquisa bibliográfica}

A análise qualitativa do material bibliográfico pesquisado foi realizada por meio de uma análise crítica interna que, segundo Marconi e Lakatos (2013), interpreta o sentido exato que o autor quis exprimir, seguindo o tratamento dos dados por intermédio de três passos sugeridos por Gil (2010): a) identificar os dados constantes dos materiais pesquisados; b) estabelecer relações dos dados obtidos com os problemas propostos; e c) analisar a consistência dos dados apresentados pelos autores.

Os dados pesquisados foram analisados e tratados com base no problema proposto, com a intenção de conseguir aporte teórico para o alcance do objetivo do estudo. Em razão disso, foi elaborado o quadro 1, um modelo teórico inicial de referência (Marconi e Lakatos, 2010), que serviu para embasar teoricamente a pesquisa documental.

\subsection{Pesquisa documental}

Na presente etapa, foram analisadas todas as informações pertinentes aos Indicadores de Gestão de Pessoas, apresentados pelos IFETs, nos relatórios de gestão dos períodos de 2009 a 2014, totalizando 228 relatórios (TCU, 2014). É importante salientar que o exercício de 2009 foi o primeiro ano de gestão dessas instituições no formato de IFETs.

A análise qualitativa do material coletado nos relatórios foi realizada por meio de análise de conteúdo (Bardin, 2011; Vergara, 2012). Assim, a referida técnica compreendeu os seguintes passos: pré-análise e organização dos documentos, exploração e tratamento dos dados e, posteriormente, análise e interpretação dos dados.

Os documentos coletados foram pré-analisados de acordo com as determinações previstas nas demandas anuais do TCU. Desse modo, pesquisou-se em cada um dos 228 relatórios de gestão coletados a presença das informações pertinentes ao item "Indicadores Gerenciais sobre Recursos Humanos", ou, na ausência dessas informações, as devidas justificativas inseridas na parte introdutória do documento.

Após os procedimentos de pré-análise, organização e exploração inicial dos dados coletados, mediante uma leitura específica sobre os conteúdos de interesse do estudo, os dados da pesquisa documental foram codificados, categorizados e, por fim, reorganizados. 
Com base na categorização proposta no quadro 3, os dados coletados nos relatórios de gestão foram reorganizados de acordo com o seu código de análise e exercício de referência.

\begin{tabular}{|c|c|}
\hline Codificação & Categorização dos dados \\
\hline A & Apresentou a existência de um ou mais Indicadores de Gestão de Pessoas. \\
\hline B & $\begin{array}{c}\text { Apresentou somente alguns dados e informações pertinentes à área } \\
\text { de Gestão de Pessoas, mas não apontou a existência de indicadores } \\
\text { utilizados para o gerenciamento dos recursos humanos da instituição. }\end{array}$ \\
\hline C & $\begin{array}{c}\text { Não apresentou a existência de indicadores utilizados para o } \\
\text { gerenciamento dos recursos humanos, mas justificou a ausência das } \\
\text { informações solicitadas no relatório. }\end{array}$ \\
\hline D & Não apresentou informações acerca de Indicadores de Gestão de Pessoas. \\
\hline
\end{tabular}

Quadro 3 - Codificação e categorização dos dados da pesquisa documental

Fonte: elaborado pelos autores (2016).

Dessa maneira, a partir dos dados codificados como "A" e "C", delimitaramse os conteúdos a serem analisados e interpretados na pesquisa documental. A categoria de análise "A", composta por 54 recortes de relatórios de gestão, abrange informações referentes aos Indicadores de Gestão de Pessoas apresentados pelos Institutos Federais. Já a categoria de análise "C", formada por 83 fragmentos de textos extraídos dos relatórios pré-analisados, compreende as principais justificativas dos IFETs para a não apresentação de indicadores da área de gestão de pessoal.

Os dados pertencentes às categorias relacionadas com os códigos de análise "B" e "D" foram descartados, pois não contemplam o objetivo do estudo. Isso porque, nos dados atribuídos à categoria de análise "B", não foram localizados "indicadores compostos", ou seja, aqueles que expressam a relação entre dois ou mais componentes de cálculo. Nos documentos coletados e classificados como código "D", não foram localizadas informações acerca da utilização de Indicadores de Gestão de Pessoas, nem ao menos as justificativas para a ausência desses indicadores.

Os procedimentos de análise e interpretação dos dados documentais foram realizados de acordo com a categorização exposta anteriormente, fundamentando-se, assim, nas teorias e objetivo do estudo. Também é importante salientar que, entre os indicadores de gestão separados para análise, a presença do "Índice de Titulação do Corpo Docente" não foi contabilizada para o presente estudo, pois trata-se de um indicador gerado anualmente pela Secretaria de Educação Profissional e Tecnológica (Setec/MEC) e apresentado separadamente nos relatórios de gestão, conforme as orientações do Acórdão 2.267/2005-TCU (TCU, 2005) e determinações das normativas do tribunal. 
Para o agrupamento e classificação dos 190 indicadores coletados e analisados, utilizou-se como base o modelo teórico inicial de referência (Quadro 1). Contudo, ao longo do processo de agrupamento, para a devida classificação dos indicadores coletados, foi necessária a inclusão de mais dois tipos que não haviam sido considerados no modelo teórico inicial, os indicadores de "Avaliação de Desempenho" e de "Disciplina".

\subsection{Pesquisa de campo}

A pesquisa de campo utilizou o método de entrevista semiestruturada como procedimento de coleta de dados. Os gestores de pessoal dos três Institutos $\mathrm{Fe}$ derais gaúchos participaram de uma entrevista por pauta, gravada e realizada com o apoio de um roteiro. A definição dos participantes considerou a sua "representatividade" (Vergara, 2009), isto é, o seu envolvimento com o objeto de estudo, proporcionando informações relevantes ao trabalho Gil (2010). Já a escolha dos três Institutos Federais gaúchos se deu em razão da "acessibilidade geográfica"(Vergara, 2013) do pesquisador às referidas sedes administrativas dos IFETs.

O roteiro de entrevista foi desenvolvido optando-se pela divisão das pautas de pesquisa em dois blocos de perguntas, a fim de melhor atender ao objetivo proposto no estudo. Dessa forma, no primeiro bloco, com a finalidade de enriquecer e aprofundar as análises e interpretações iniciadas na pesquisa documental, definiram-se as pautas apresentadas no quadro 4.

\begin{tabular}{|c|c|}
\hline Pautas 1e 2 (Bloco I) & Questões do roteiro de entrevistas \\
\hline $\begin{array}{l}\text { 1- Atual situação dos } \\
\text { Institutos Federais no } \\
\text { processo de aplicação } \\
\text { de Indicadores de } \\
\text { Gestão de Pessoas. }\end{array}$ & $\begin{array}{l}\text { - Atualmente, o instituto trabalha com Indicadores de Ges- } \\
\text { tão de Pessoas? Comente sobre a atual utilização e/ou de- } \\
\text { senvolvimento destes indicadores na instituicão. } \\
\text { - Você considera que a utilização de Indicadores de Gestão } \\
\text { de Pessoas como uma ferramenta de gestão é importante } \\
\text { para os Institutos Federais? Se sim, quais seriam os possí- } \\
\text { veis benefícios dessa ferramenta? }\end{array}$ \\
\hline $\begin{array}{l}2 \text { - Dificuldades } \\
\text { encontradas pelos } \\
\text { Institutos Federais } \\
\text { para a utilização de } \\
\text { indicadores da área de } \\
\text { gestão de pessoal. }\end{array}$ & $\begin{array}{l}\text { - Atualmente, o instituto encontra alguma dificuldade na } \\
\text { utilização e/ou desenvolvimento de indicadores da área de } \\
\text { gestão de pessoas? Se sim, comente sobre as dificuldades } \\
\text { encontradas. } \\
\text { - Você acredita que a inexistência de um modelo padro- } \\
\text { nizado de Indicadores de Gestão de Pessoas dificulta a } \\
\text { utilização desses indicadores nos Institutos Federais? Por } \\
\text { quê? }\end{array}$ \\
\hline
\end{tabular}

Quadro 4 - Pauta das entrevistas do Bloco I

Fonte: elaborado pelos autores (2016). 
O segundo bloco de perguntas, apresentado no quadro 5, foi empregado para avaliar o modelo de Indicadores de Gestão de Pessoas desenvolvido após os resultados das pesquisas bibliográfica e documental. Assim, utilizaram-se questionamentos relativos ao atendimento dos requisitos essenciais dos indicadores de gestão e à utilidade e aplicação prática do modelo proposto, de modo a assegurar a relevância dos indicadores, por meio da validação do modelo apresentado.

\begin{tabular}{|c|c|c|}
\hline \multicolumn{3}{|c|}{ Pauta 3 (Bloco II): Avaliação do modelo de Indicadores de Gestão de Pessoas } \\
\hline Codificação & $\begin{array}{l}\text { Categorias de } \\
\text { análise }\end{array}$ & Questões do roteiro de entrevistas \\
\hline \multirow{3}{*}{$\begin{array}{l}\text { Requisitos } \\
\text { essenciais } \\
\text { recomendados }\end{array}$} & Validade & $\begin{array}{l}\text { - Todos os indicadores do modelo refletem ade- } \\
\text { quadamente a realidade que se deseja medir? } \\
\text { (Algum dos indicadores não pode ser aferido } \\
\text { pela respectiva fórmula de cálculo proposta?) }\end{array}$ \\
\hline & Confiabilidade & $\begin{array}{l}\text { - Todos os indicadores do modelo possuem com- } \\
\text { ponentes de cálculo com fontes de dados confi- } \\
\text { áveis e atualizadas? (Algum dos indicadores não } \\
\text { possui fonte de dados adequada para aferir o } \\
\text { seu resultado?) }\end{array}$ \\
\hline & Simplicidade & $\begin{array}{l}\text { - Todos os indicadores do modelo podem ser fa- } \\
\text { cilmente compreendidos pelo público em geral? } \\
\text { (Algum dos indicadores aparenta ser de difícil } \\
\text { compreensão?) }\end{array}$ \\
\hline \multirow{3}{*}{$\begin{array}{l}\text { Utilidade do } \\
\text { modelo proposto }\end{array}$} & Tomada de decisão & $\begin{array}{l}\text { - Você acredita que a aplicação prática do mo- } \\
\text { delo apresentado pode auxiliar os gestores na } \\
\text { tomada de decisão sobre a gestão de pessoas? } \\
\text { Por quê? }\end{array}$ \\
\hline & $\begin{array}{l}\text { Transparência de } \\
\text { informações }\end{array}$ & $\begin{array}{l}\text { - A aplicação prática do modelo apresentado po- } \\
\text { deria facilitar a transparência de informações à } \\
\text { sociedade e também aos órgãos controladores? } \\
\text { Justifique a sua resposta. }\end{array}$ \\
\hline & Aplicação prática & $\begin{array}{l}\text { - Você acredita que o modelo apresentado pode- } \\
\text { ria ser aplicado na sua instituição? Por quê? }\end{array}$ \\
\hline
\end{tabular}

Quadro 5 - Pauta das entrevistas do Bloco II

Fonte: elaborado pelos autores (2016).

A validação é um dos pontos fortes da pesquisa qualitativa, pois consiste em determinar se os resultados são adequados do ponto de vista dos participantes. De acordo com Paiva Jr., Leão e Mello (2011), o feedback dos envolvidos representa uma validação comunicativa, correspondente à obtenção de sua concordância ou aceitação. Tal procedimento pode envolver a realização de entrevistas de acompanhamento com os participantes do estudo, proporcionando uma oportunidade para eles comentarem os resultados Creswell (2010). 
Inicialmente, para a realização das entrevistas, os gestores de pessoal dos três Institutos Federais gaúchos foram contatados via telefone e e-mail. Nesse primeiro contato, eles foram convidados a participar da pesquisa, ressaltando-se a importância da participação do gestor como entrevistado e apresentando-se a finalidade do estudo. No segundo contato, após o aceite dos gestores, as datas para a aplicação das entrevistas foram agendadas e o roteiro de perguntas semiestruturadas foi enviado previamente, com o intuito de familiarizar o respondente com os conceitos e delimitações definidas para o objeto de estudo, com as instruções pertinentes aos questionamentos e, principalmente, com o modelo de Indicadores de Gestão de Pessoas proposto para avaliação.

A coleta de campo ocorreu no mês de outubro de 2015, e as entrevistas tiveram um tempo médio de 56 minutos e foram transcritas logo após o término de cada coleta de campo. O quadro 6 apresenta informações sobre as entrevistas realizadas.

\begin{tabular}{|c|c|c|c|c|}
\hline Instituição & $\begin{array}{c}\text { Local da } \\
\text { entrevista }\end{array}$ & $\begin{array}{c}\text { Data da } \\
\text { entrevista }\end{array}$ & $\begin{array}{c}\text { Duração da } \\
\text { entrevista }\end{array}$ & $\begin{array}{c}\text { Denominação do } \\
\text { entrevistado }\end{array}$ \\
\hline IF Farroupilha & Santa Maria/RS & $29 / 10 / 2015$ & $71 \mathrm{~min}$ & Entrevistado 1(E1) \\
\hline IFRS & $\begin{array}{c}\text { Bento } \\
\text { Gonçalves/RS }\end{array}$ & $21 / 10 / 2015$ & $41 \mathrm{~min}$ & Entrevistado 2(E2) \\
\hline IF Sul & Pelotas/RS & $27 / 10 / 2015$ & $56 \mathrm{~min}$ & Entrevistado 3(E3) \\
\hline
\end{tabular}

Quadro 6 - Informações sobre a realização das entrevistas

Fonte: elaborado pelos autores (2016).

Dessa forma, a pesquisa de campo teve como foco principal a exposição do modelo de Indicadores de Gestão de Pessoas desenvolvido ao longo do estudo, com o intuito de avaliar, junto aos gestores de pessoal dos IFETs, se determinados indicadores são válidos para contribuir com a tomada de decisões e facilitar a transparência de informações.

\section{ANÁLISE DOS RESULTADOS}

Com relação aos Indicadores de Gestão de Pessoas utilizados pelos Institutos Federais, desde a instituição da Rede Federal EPCT, constatou-se uma evolução percentual no número de IFETs que apresentaram um ou mais indicadores no período de 2009 a 2014. A evolução percentual pode ser visualizada na figura 1. 
Figura 1 - Evolução histórica da utilização de Indicadores de Gestão de Pessoas

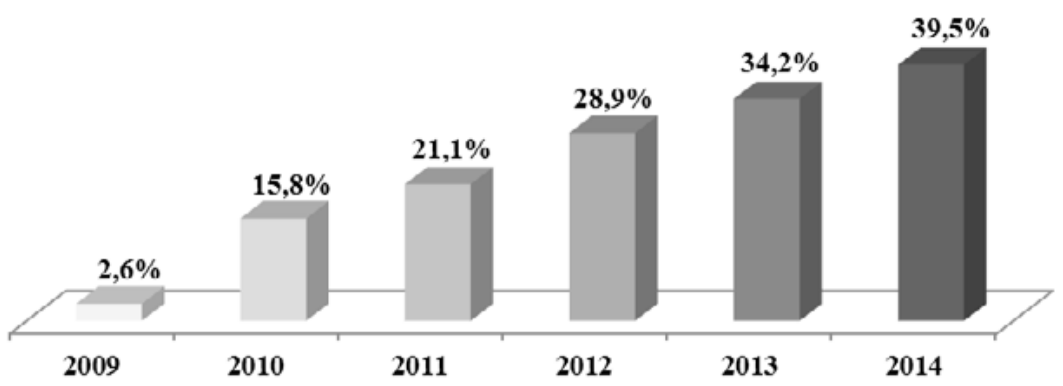

Fonte: base de dados do TCU: Contas e Relatórios de Gestão.

Após a análise inicial sobre os Indicadores de Gestão de Pessoas utilizados, verificou-se também a representatividade quantitativa e percentual dos 190 indicadores coletados: Capacitação (37; 19,5\%); Rotatividade (30; 15,8\%); Qualificação (28; 14,7\%); Absenteísmo (18; 9,5\%); Acidentes e doenças ocupacionais (17; 8,9\%); Força de trabalho (16; 8,4\%); Afastamento (13; 6,8\%); Aposentadoria (10; 5,3\%); Disciplina (9; 4,7\%); Avaliação de Desempenho (6; 3,2\%); Idade e antiguidade (3; 1,6\%); e Remuneração (3; 1,6\%).

A representatividade percentual, que indica uma ordem de interesse por parte das unidades pesquisadas, pode ser visualizada na figura 2 .

Figura 2 - Representatividade percentual dos indicadores apresentados pelos IFETs

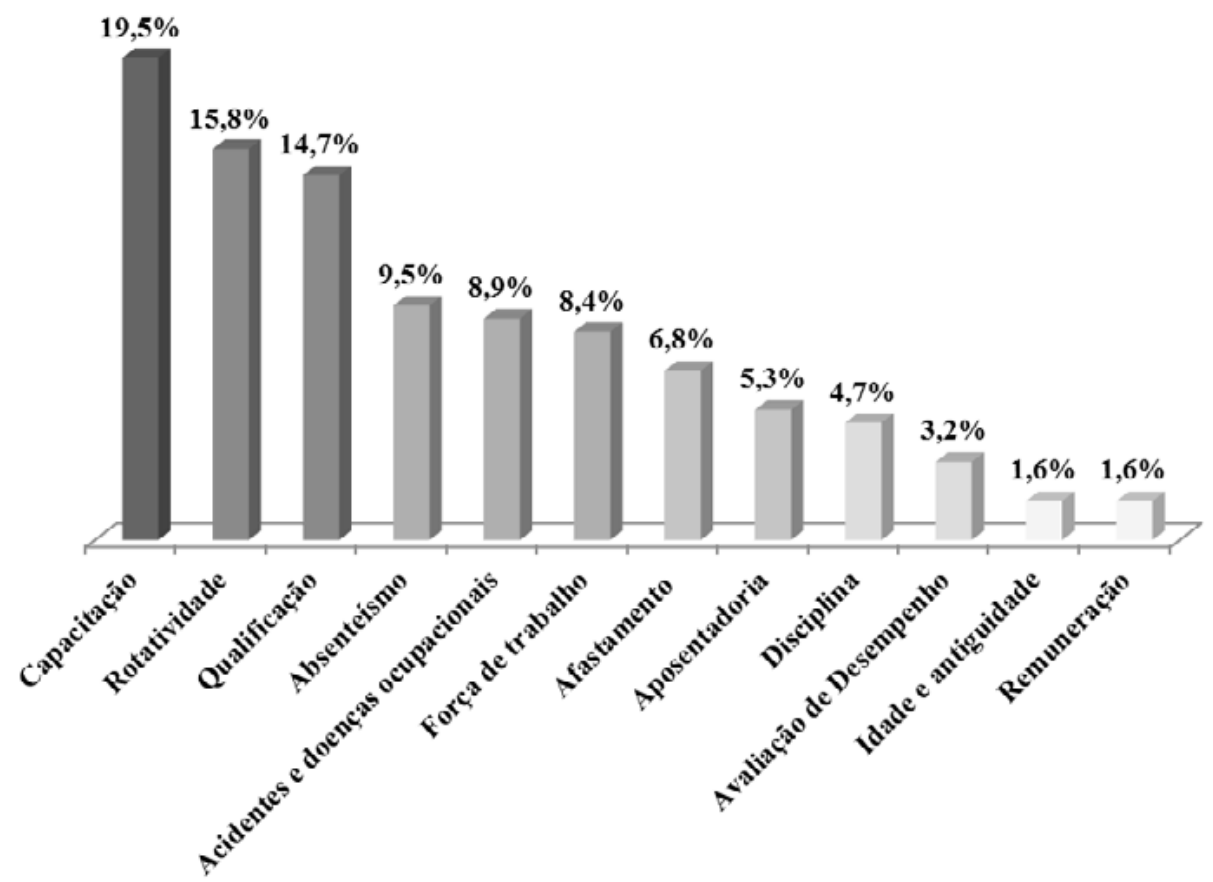

Fonte: base de dados do TCU: Contas e Relatórios de Gestão. 
A respeito do último exercício analisado, com base nos relatórios de gestão de 2014, constatou-se que: 15 Institutos Federais já possuem um modelo de Indicadores de Gestão de Pessoas contendo um ou mais indicadores; quatro IFETs estão em processo de desenvolvimento e/ou implantação de indicadores; outros quatro aguardam o desfecho da implantação de um Sistema Integrado de Gestão (SIG) para trabalhar com indicadores da área de Gestão de Pessoas; e 15 Institutos Federais ainda não fazem uso de tais indicadores e também não apresentaram previsão para desenvolvê-los. Dessa maneira, embora existam alguns avanços no número de Institutos Federais que aplicam indicadores da área de Gestão de Pessoas, verificou-se que ainda não há uma evolução satisfatória nesse processo de aplicação, visto que a maioria das instituições pesquisadas não utiliza tais indicadores e apresenta dificuldades para implantá-los.

Com relação às principais dificuldades encontradas para a utilização de Indicadores de Gestão de Pessoas por parte dos IFETs, que ratificam a incipiência do tema pesquisado, destacam-se: a) escassez de servidores (número insuficiente de pessoal); b) ausência de um sistema informatizado (ou de mecanismos apropriados); c) priorização de outras atividades (em detrimento da geração dos indicadores); e d) desinteresse por parte da gestão (principais usuários).

\subsection{Desenvolvimentode um modelo de Indicadores de Gestão de Pessoas}

Inicialmente, para a formatação do modelo, utilizaram-se os dados coletados e analisados nas pesquisas bibliográfica e documental. Após o processo de agrupamento e classificação dos Indicadores de Gestão de Pessoas estudados, de acordo com a representatividade percentual de cada tipo de métrica apresentada, verificou-se a ordem de preferência/utilização por parte das instituições pelos indicadores mais utilizados no período de 2009 a 2014.

Com o intuito de formatar um modelo contendo apenas os indicadores mais relevantes - de maior preferência - para os Institutos Federais, evitando a criação de métricas em demasia, os indicadores dos tipos "Idade e antiguidade" e "Remuneração", que apresentaram os menores índices de utilização entre os IFETs, foram descartados do modelo.

Logo depois, durante a definição das métricas que iriam compor o modelo, verificou-se ainda a necessidade de exclusão dos indicadores de "Avaliação de Desempenho" e de "Disciplina", pois, além de também apresentarem pouca preferência - aplicação prática - por parte dos Institutos Federais, não foi encontrado na etapa de pesquisa bibliográfica o respaldo teórico necessário para propor métricas para tais tipos de indicadores. 
Dessa forma, o desenvolvimento do modelo de Indicadores de Gestão de Pessoas se deu a partir de oito tipos de indicadores. Em seguida, para a definição das métricas que compõem o modelo, consideraram-se as informações das pesquisas bibliográfica (respaldo teórico) e documental (aplicação na prática), conforme descrito no quadro 7 .

\begin{tabular}{|c|c|c|c|}
\hline $\begin{array}{l}\text { Tipo de } \\
\text { indicador }\end{array}$ & $\begin{array}{l}\text { Indicadores } \\
\text { propostos }\end{array}$ & Descrição & Fórmula de cálculo \\
\hline Absenteísmo & $\begin{array}{l}\text { 1) Índice de } \\
\text { absenteísmo }\end{array}$ & $\begin{array}{l}\text { Demonstra o nível } \\
\text { de abstenção de } \\
\text { servidores ao trabalho. }\end{array}$ & $\begin{array}{l}\text { Total de dias não } \\
\text { trabalhados } /\left(n^{\circ} \text { total de }\right. \\
\text { servidores * } n^{\circ} \text { total de } \\
\text { dias úteis }) * 100\end{array}$ \\
\hline $\begin{array}{l}\text { Acidentes } \\
\text { de trabalho } \\
\text { e doenças } \\
\text { ocupacionais }\end{array}$ & $\begin{array}{l}\text { 2) Índice de } \\
\text { acidentes } \\
\text { e doenças } \\
\text { ocupacionais }\end{array}$ & $\begin{array}{l}\text { Indica o percentual } \\
\text { médio de acidentes de } \\
\text { trabalho e casos de } \\
\text { doenças ocupacionais } \\
\text { por colaborador. }\end{array}$ & $\begin{array}{l}\left(N^{\circ} \text { total de acidentes }\right. \\
+n^{\circ} \text { total de doenças } \\
\text { ocupacionais) / } n^{\circ} \text { total } \\
\text { de servidores * } 100\end{array}$ \\
\hline Afastamento & $\begin{array}{l}\text { 3) Índice de } \\
\text { afastamento }\end{array}$ & $\begin{array}{c}\text { Apresenta o percentual } \\
\text { de servidores afastados } \\
\text { do trabalho. }\end{array}$ & $\begin{array}{c}N^{\circ} \text { de servidores } \\
\text { licenciados ou com } \\
\text { afastamento legal / } n^{\circ} \\
\text { total de servidores * } 100\end{array}$ \\
\hline \multirow[b]{2}{*}{ Aposentadoria } & $\begin{array}{l}\text { 4) Previsão de } \\
\text { aposentadoria }\end{array}$ & $\begin{array}{l}\text { Indica o percentual } \\
\text { de servidores com } \\
\text { previsão legal de } \\
\text { aposentadoria. } \\
\end{array}$ & $\begin{array}{c}N^{\circ} \text { de servidores } \\
\text { com previsão de } \\
\text { aposentadoria } / n^{\circ} \text { total } \\
\text { de servidores * } 100\end{array}$ \\
\hline & $\begin{array}{l}\text { 5) Reposição de } \\
\text { aposentadoria }\end{array}$ & $\begin{array}{l}\text { Verifica o percentual } \\
\text { de ocupação de } \\
\text { vagas originadas de } \\
\text { aposentadoria via } \\
\text { ingresso de novos } \\
\text { servidores. } \\
\end{array}$ & $\begin{array}{c}N^{\circ} \text { de provimentos de } \\
\text { vagas de aposentadorias } \\
/ n^{\circ} \text { de servidores } \\
\text { aposentados no período } \\
* 100\end{array}$ \\
\hline Capacitação & $\begin{array}{l}\text { 6) Índice de } \\
\text { capacitação }\end{array}$ & $\begin{array}{l}\text { Mede o percentual de } \\
\text { servidores capacitados. }\end{array}$ & $\begin{array}{c}N^{\circ} \text { de servidores } \\
\text { capacitados } / n^{\circ} \text { total de } \\
\text { servidores }{ }^{*} 100\end{array}$ \\
\hline $\begin{array}{l}\text { Força de } \\
\text { trabalho }\end{array}$ & $\begin{array}{l}\text { 7) Variação } \\
\text { no quadro de } \\
\text { servidores }\end{array}$ & $\begin{array}{c}\text { Indica o nível de } \\
\text { aumento ou redução do } \\
\text { quantitativo do quadro } \\
\text { de servidores. }\end{array}$ & $\begin{array}{c}{\left[\left(N^{\circ} \text { total de servidores }\right.\right.} \\
/ n^{\circ} \text { total de servidores } \\
\text { no período anterior })-1] \\
* 100\end{array}$ \\
\hline Qualificação & $\begin{array}{l}\text { 8) Nível de } \\
\text { qualificação }\end{array}$ & $\begin{array}{c}\text { Apresenta o atual } \\
\text { índice de titulação dos } \\
\text { servidores. }\end{array}$ & $\begin{array}{c}N^{\circ} \text { de servidores por } \\
\text { nível de qualificação / } n^{\circ} \\
\text { total de servidores }\end{array}$ \\
\hline Rotatividade & $\begin{array}{l}\text { 9) Índice de } \\
\text { rotatividade }\end{array}$ & $\begin{array}{l}\text { Mede o giro de } \\
\text { entradas e saídas de } \\
\text { servidores. }\end{array}$ & $\begin{array}{c}{\left[\left(N^{\circ} \text { de entradas de }\right.\right.} \\
\text { novos servidores }+n^{\circ} \text { de } \\
\text { saídas de servidores) / } \\
2] / n^{\circ} \text { total de servidores } \\
* 100\end{array}$ \\
\hline
\end{tabular}

Quadro 7 - Proposta de modelo de Indicadores de Gestão de Pessoas

Fonte: elaborado pelos autores (2016). 
Com a intenção de verificar se os indicadores propostos atendem às demandas dos gestores de pessoal dos IFETs (IF Farroupilha: E1; IFRS: E2; IF Sul: E3), o modelo composto por nove indicadores foi avaliado considerando o atendimento aos requisitos essenciais recomendados, a possibilidade de aplicação prática dos indicadores e a contribuição com a tomada de decisões gerenciais e a transparência de informações à sociedade e aos órgãos controladores.

Sobre os requisitos dos indicadores apresentados, a partir dos comentários e opiniões dos gestores frente ao atendimento dos três requisitos essenciais de validade, confiabilidade e simplicidade, realizou-se uma análise individual de cada indicador avaliado. O quadro 8 apresenta a síntese dos resultados da avaliação sobre os indicadores do modelo proposto.

\begin{tabular}{|c|c|c|c|c|c|c|c|c|c|}
\hline \multirow{2}{*}{$\begin{array}{l}\text { Avaliação do modelo } \\
\text { de Indicadores de } \\
\text { Gestão de Pessoas }\end{array}$} & \multicolumn{3}{|c|}{ Validade } & \multicolumn{3}{|c|}{ Confiabilidade } & \multicolumn{3}{|c|}{ Simplicidade } \\
\hline & E1 & E2 & E3 & E1 & E2 & E3 & E1 & E2 & E3 \\
\hline Índice de absenteísmo & $\checkmark$ & $\checkmark$ & $\checkmark$ & - & - & $\checkmark$ & - & $\checkmark$ & $\checkmark$ \\
\hline $\begin{array}{l}\text { Índice de acidentes e } \\
\text { doenças ocupacionais }\end{array}$ & $\checkmark$ & $\checkmark$ & $\checkmark$ & $\checkmark$ & - & $\checkmark$ & $\checkmark$ & $\checkmark$ & $\checkmark$ \\
\hline Índice de afastamento & $\checkmark$ & $\checkmark$ & $\checkmark$ & $\checkmark$ & $\checkmark$ & $\checkmark$ & $\checkmark$ & $\checkmark$ & $\checkmark$ \\
\hline $\begin{array}{c}\text { Previsão de } \\
\text { aposentadoria }\end{array}$ & $\checkmark$ & $\checkmark$ & - & - & $\checkmark$ & $\checkmark$ & $\checkmark$ & $\checkmark$ & $\checkmark$ \\
\hline $\begin{array}{l}\text { Reposição de } \\
\text { aposentadoria }\end{array}$ & $\checkmark$ & $\checkmark$ & $\checkmark$ & $\checkmark$ & $\checkmark$ & $\checkmark$ & $\checkmark$ & $\checkmark$ & $\checkmark$ \\
\hline Índice de capacitação & $\checkmark$ & $\checkmark$ & $\checkmark$ & $\checkmark$ & $\checkmark$ & $\checkmark$ & $\checkmark$ & $\checkmark$ & $\checkmark$ \\
\hline $\begin{array}{l}\text { Variação no quadro de } \\
\text { servidores }\end{array}$ & $\checkmark$ & $\checkmark$ & $\checkmark$ & $\checkmark$ & $\checkmark$ & $\checkmark$ & $\checkmark$ & $\checkmark$ & $\checkmark$ \\
\hline Nível de qualificação & $\checkmark$ & $\checkmark$ & $\checkmark$ & $\checkmark$ & $\checkmark$ & $\checkmark$ & $\checkmark$ & $\checkmark$ & $\checkmark$ \\
\hline Índice de rotatividade & $\checkmark$ & $\checkmark$ & $\checkmark$ & $\checkmark$ & - & $\checkmark$ & - & $\checkmark$ & - \\
\hline
\end{tabular}

Quadro 8 - Extrato dos resultados da avaliação sobre os indicadores do modelo proposto Fonte: elaborado pelos autores (2016).

Conforme pode ser visualizado no quadro 8 , considerando os requisitos essenciais recomendados, os indicadores do modelo foram bem avaliados pelos gestores entrevistados. Todavia, com relação a esses requisitos, quatro dos nove indicadores propostos não tiveram aprovação unânime, demandando, assim, maiores cuidados para uma possível aplicação prática.

Posteriormente, verificou-se a utilidade do modelo de Indicadores de Gestão de Pessoas proposto, no que tange às seguintes categorias de análise: tomada de decisão, transparência de informações e aplicação prática.

Conforme analisado nas falas dos entrevistados, os gestores E2 e E3 entendem que o modelo proposto pode auxiliar os usuários na tomada de decisão sobre 
a gestão de pessoas. Para os gestores, trata-se de um "modelo bem prático"(E3), que possibilita "tomar decisões para resolver os problemas existentes"(E2). Entretanto, o gestor E1 alerta sobre a necessidade da percepção de utilidade por parte de todos os envolvidos, pois"[...] não adianta apenas aplicar um modelo sem que o grupo de pessoas compreenda e trabalhe para constituí-Io".

Com relação à transparência de informações, de acordo com as opiniões coletadas, os três entrevistados consideram que o modelo proposto pode facilitar a transparência de informações. O gestor E2 explica que a Gestão de Pessoas lida com frequência com os órgãos de controle, "[...] o que eles querem é justamente enxergar as ferramentas que tu utiliza". Segundo E3, a aplicação do modelo apresentado "expõe ao público interessado muitas informações sobre o quadro de servidores de determinada instituição".

Por fim, os entrevistados concordam que o modelo proposto pode ser aplicado nas instituições em que atuam, mas talvez não na sua totalidade, pois, segundo os gestores, determinados indicadores podem ser de difícil aferição, por conta da ausência de alguns dados necessários para compor uma ou mais métricas.

Assim sendo, a partir das respostas dos entrevistados sobre a utilidade do modelo apresentado, foi possível avaliá-lo de forma positiva, visto que os gestores consideram que a maior parte das métricas propostas possui aplicação prática imediata e que o modelo pode plenamente contribuir com a tomada de decisões gerenciais e facilitar a transparência de informações à sociedade e aos órgãos controladores.

\section{CONSIDERAÇÕES FINAIS}

Com base na concretização de todas as etapas previstas na pesquisa, entende-se que o objetivo central do estudo, "desenvolver um modelo de Indicadores de Gestão de Pessoas para Institutos Federais de Educação, Ciência e Tecnologia", foi substancialmente alcançado. Contudo, cabe ressaltar que, por conta da possível ausência de alguns dados necessários para aferir determinados indicadores apresentados, provavelmente, nem todos os Institutos Federais tenham condições de aplicar integralmente o modelo proposto.

Ademais, ao término deste estudo, foram percebidas duas limitações na pesquisa realizada. A primeira é referente à fonte de dados utilizada para a pesquisa documental, visto que foram utilizados apenas os relatórios de gestão do banco de dados do TCU para a coleta dos indicadores analisados, desconsiderando a possível existência de indicadores de gestão não tornados públicos pelas instituições. Outra limitação foi o reduzido número de entrevistas realizadas, justificado pela dificuldade de acessibilidade geográfica. 
Para pesquisas futuras, considerando as diversas dificuldades encontradas pelos Institutos Federais na utilização dos indicadores analisados, sugere-se a realização de estudos envolvendo casos de sucesso quanto à aplicação de métricas como uma ferramenta de gestão no setor público. Dessa forma, as implicações dessas pesquisas poderiam motivar outras instituições a adotar sistemas de medições com maior intensidade na administração pública.

De forma geral, considerando que os indicadores do modelo foram bem avaliados e aceitos pelos gestores entrevistados, entende-se que o resultado deste estudo possui um potencial de aplicação prática imediata. Igualmente, é importante ressaltar que o conjunto de indicadores apresentado pode contribuir com a tomada de decisões gerenciais e facilitar a transparência de informações à sociedade e aos órgãos controladores.

Por fim, espera-se que os resultados deste estudo possam estimular gestores e instituições a trabalhar com ferramentas de apoio à decisão, que visem promover o controle, facilitar o planejamento e fortalecer os mecanismos de transparência da organização.

\section{REFERÊNCIAS}

Amato Neto, J. (2009). Gestão de Sistemas Locais de Produção e Inovação. São Paulo: Atlas.

Assis, M. T. (2012). Indicadores de Gestão de Recursos Humanos (2. ed.). Rio de Janeiro: Qualitymark.

Bardin, L. (2011). Análise de conteúdo. São Paulo: Edições 70.

Bergue, S. T. (2010). Gestão de pessoas em organizações públicas (3. ed.). Caxias do Sul: EDUCS.

Bergue, S. T. (2014). Gestão estratégica de pessoas no setor público. São Paulo: Atlas.

Caldeira, J. (2012). 100 Indicadores da Gestão. Lisboa: Actual.

Carlson, K. D., \& Kavanagh, M. J. (2012). HR metrics and workforce analytics. In M. J. Kavanagh, M. Thite, \&R. D. Johnson (Eds), Human Resource Information Systems: Basics applications and future directions. Thousand Oaks, CA: Sage Publishing.

Chaves, R. S. (2011). Auditoria e controladoria no setor público: Fortalecimento dos controles internos (2. ed.). Curitiba: Juruá.

Costa, N. P. (2010). Análise do resultado empresarial: Principais ferramentas da análise corporativa. Rio de Janeiro: Ciência Moderna. 
Creswell, J. W. (2010). Projeto de pesquisa: Métodos qualitativo, quantitativo e misto (3. ed.). Porto Alegre: Artmed/Bookman.

Fitz-enz, J. (1984). How to measure Human Resources management. New York: Mc Graw-Hill Inc.

Gil, A.C. (2010). Como elaborar projetos de pesquisa (5. ed.). São Paulo: Atlas.

Jaramillo, J.M.B. (2003). Indicadores de Gestión: Herramientas para lograr la competitividad (2. ed.). Bogotá: 3R Panamericana Editorial.

LeGall, J. (2008). Gestão de recursos humanos. São Paulo: Ática.

Marconi, M.A., \& Lakatos, E. M. (2010). Fundamentos da metodologia científica (7. ed.). São Paulo: Atlas.

Marconi, M. A., \& Lakatos, E. M. (2013). Metodologia do trabalho científico (7. ed.). São Paulo: Atlas.

Milioni, B. (2005). Indicadores para gestão de treinamento e desenvolvimento. São Paulo: ABTD.

Paiva Jr., F. G., Leão, A. L. M. S., \& Mello, S. C. B. (2011). Validade e confiabilidade na pesquisa qualitativa em Administração. Revista de Ciências da Administração, 13, (31), 190-209.

Pavani Jr. O., \& Scucuglia, R. (2011). Mapeamento e Gestão por Processos. São Paulo: M. Books.

Pomi, R. M. (2002). Indicadores de Desempenho em Gestão do Capital Humano. In BOOG, G.; BOOG, M. (Coord.), Manual de gestão de pessoas e equipes: Estratégias e Tendências (Vol. 1). São Paulo: Gente.

Takashina, N. T.; Flores, M. C. X. (1996). Indicadores da qualidade e do desempenho: Como estabelecer metas e medir resultados. Rio de Janeiro: Qualitymark.

Tachizawa, N. T., Ferreira, V. C. P., Fortuna, \& Antônio, A. M. (2006). Gestão com pessoas: Uma abordagem aplicada às estratégias de negócios (5. ed). Rio de Janeiro: FGV.

TCU. Tribunal de Contas da União. (2005). Acórdão 2.267-TCU/2005: Método de cálculo para indicadores de Gestão da Rede Federal de Educação Profissional e Tecnológica - EPT. Recuperado de http://portal.mec.gov.br/setec/ arquivos/pdf/indicadores_gestao_maio.pdf

TCU. Tribunal de Contas da União. (2014). Relatórios de Gestão, Decisões Normativas e demais Portarias: Exercícios 2014-13-12-11-10-09. Recuperado de http://portal.tcu.gov.br/contas/contas-e-relatorios-de-gestao/contas-do -exercicio-de-2014.htm 
Vergara, S. C. (2009). Métodos de coleta de dados no campo. São Paulo: Atlas.

Vergara, S. C. (2012). Métodos de pesquisa em administração (5. ed.). São Paulo: Atlas.

Vergara, S.C. (2013). Projetos e relatórios de pesquisa em administração (14. ed.). São Paulo: Atlas.

Yin, R. K. (2015). Estudo de casos: Planejamento e métodos (5. ed.) Porto Alegre: Bookman. 